\title{
AVALIAÇÃO DE ÍNDICES DE DEPENDÊNCIA ESPACIAL DE MODELOS GEOESTATÍSTICOS SOBRE A KRIGAGEM
}

Luiz Otávio Rodrigues Pinto', Natielle Gomes Cordeiro², Rafael Lucas Figueiredo de Souza $^{3}$, Rafaella Tavares Pereira ${ }^{4}$, José Márcio de Mello ${ }^{5}$

1Doutorando em Engenharia Florestal pela Universidade Federal de Lavras - UFLA, Lavras, Minas Gerais, Brasil. E-mail: luizorp@outlook.com

2 Doutoranda em Engenharia Florestal pela Universidade Federal de Lavras - UFLA

${ }^{3}$ Mestrando em Engenharia Florestal pela Universidade Federal de Lavras - UFLA

${ }^{4}$ Graduanda em Engenharia Florestal pela Universidade Federal de Lavras - UFLA

${ }^{5}$ Professor Doutor Adjunto da Universidade Federal de Lavras - UFLA

Recebido em: 06/04/2019 - Aprovado em: 10/06/2019 - Publicado em: 30/06/2019 DOI: 10.18677/EnciBio_2019A25

\begin{abstract}
RESUMO
O semivariograma é utilizado na geoestatística para a estimativa do grau de dependência espacial, inferindo sobre a relação de uma variável espacializada. Atualmente existem metodologias que utilizam com mais eficiência os parâmetros do semivariograma e, a escolha do melhor método é fundamental para a seleção de modelos para a krigagem. O objetivo do trabalho foi avaliar índices de dependência espacial para a seleção de modelos teóricos sobre a krigagem. Os dados são provenientes de povoamentos clonais de Eucalyptus sp. em três regiões de Minas Gerais. Foram ajustados os modelos esférico e exponencial para o volume, visando obter o conjunto de parâmetros para as respectivas funções. Para todos os ajustes foram classificados a estrutura de continuidade espacial pelos métodos do GDE e IDE. Aproximadamente $60 \%$ dos ajustes apresentaram forte dependência espacial pelo método $\mathrm{DE}$, enquanto aproximadamente $50 \%$ apresentaram classificação forte pelo IDE. O índice DE classificou 117 ajustes como forte dependência espacial, sendo que pelo novo índice (IDE) 40\% mudaria a classificação de forte para fraca.

Quando comparado os métodos de ajuste mínimos quadrados e máxima verossimilhança, houve alterações em $28 \%$ das análises. Apesar dos mapas de krigagem apresentarem alta correlação observou-se a diferença de área das classes volumétricas e consequentemente o volume médio. Usando uma base de dados robusta e mais informações do semivariograma, o uso do IDE para a seleção de modelos se mostrou mais eficiente. Assim este é recomendado para descrever a dependência espacial, por contemplar todos os parâmetros do semivariograma e fatores de correção para cada modelo.
\end{abstract}

PALAVRAS-CHAVE: Continuidade espacial; geoestatistica;Modelagem. 


\title{
EVALUATION OF SPATIAL DEPENDENCE INDEXES OF GEOSTATISTICAL MODELS ABOUT THE KRIGING
}

\begin{abstract}
The semivariogram is used in geostatistics to predict the degree of spatial dependence, inferring about the relation of a spatialized variable. Nowadays there are methodologies that use with more efficiency the semivariogram parameters and the choice of method is important for selecting models for kriging. The aim of this study was to evaluate spatial dependence index to the selection of theoretical models to Kriging. The data were obtained from clonal stands of Eucalyptus sp. in three regions of Minas Gerais. Spherical and exponential models were fitted to volume, looking for obtain sets of parameters for the functions. To all adjustment were classified the structure of spatial continuity by using the methods GDE and IDE. Approximately $60 \%$ of the adjustments were classified as strong spatial dependence by the GDE method, while approaching $50 \%$ showed classification as strong by the method IDE. The GDE index ranked 117 adjustment as strong spatial dependece, being that by the new index (SDI) $40 \%$ would change the classification from strong to weak. When comparing the methods of least square adjustment and maximum likelihood, there were alterations in $28 \%$ of the analyzes. Although the kriging maps show high correlation, it was possible to observe the difference of area to the volumetric classes and consequently the mean volume. Using a robust database and more information from the semivariogram, the IDE showed to be more efficient to selection of models. Thus it is recommended to describe a spatial dependence because it includes all semivariogram parameters and correction factors for each model.
\end{abstract}

KEYWORDS: Geostatistics, Space Continuity, Modeling.

\section{INTRODUÇÃO}

A estimativa do estoque presente permite inferir quanto ao crescimento e desenvolvimento de uma determinada área e consequentemente fornece subsídios para a tomada de decisão a ser realizada (BINOTI et al., 2014). Assim, o volume de madeira se caracteriza por ser uma variável de suma importância nos quesitos operacionais e estratégicos (OLIVEIRA et al., 2014). No entanto, a quantificação deste atributo requer de procedimentos minuciosos, por vezes demorados e onerosos, que são intrinsecamente dependentes da intensidade amostral (LUNDGREN et al., 2015).

O procedimento usual e difundido na área florestal para a estimativa do estoque volumétrico é a utilização de modelos matemáticos que consideram a relação entre o volume e variáveis como diâmetro e altura. No entanto, buscando a otimização na estimativa da característica de interesse, técnicas como as redes neurais artificiais, sensoriamento remoto e a estatística espacial (BINOTI et al., 2014; GORGENS et al., 2014; BARROS et al., 2015; LEAL et al., 2015; LUNDGREN et al., 2015) são empreendidas.

Lundgren et al. (2016) afirmam que a geoestatística vem sendo utilizada como um método auxiliar nas ciências florestais, favorecendo uma possível diminuição no custo relativo do inventário, além de fornecer resultados mais detalhados bem como confiáveis quanto aos obtidos por meio do uso da estatística clássica. Ademais, cabe destacar o quão notório é a necessidade do reconhecimento e quantificação da variabilidade espacial da produtividade nas florestas (SANTOS et al., 2017). Assim, 
na Geoestatística a análise da dependência espacial é realizada por meio do semivariograma, ferramenta que viabiliza a observação da estrutura de dependência espacial e consiste em um gráfico que associa distâncias com semivariâncias (SEIDEL; OLIVEIRA., 2014b).

Por meio do semivariograma é possível obter parâmetros como efeito pepita (nugget) que está atrelado ao erro não amostral, a contribuição (partial sill) que é onde a geoestatística atua, detectando a influência da distância sobre a variância da variável estudada e o alcance que é compreendido como a distância dentro da qual as amostras apresentam-se correlacionadas espacialmente (range) (ACERBI JÚNIOR et al., 2015). Estes parâmetros permitem a caracterização da dependência espacial que expressa o comportamento da correlação entre dados observados, sendo essencial em análises geoestatísticas pois possibilita o ajuste de modelos matemáticos. Além disso, é possível por meio destes expressar o grau e a magnitude da estrutura de continuidade espacial (MELLO et al., 2008; ODA-SOUZA et al., 2008).

Dentre as metodologias para definição do grau de dependência espacial podem ser destacados as propostas por Biondi et al., (1994) e Cambardella et al. (1994). Esses métodos de avaliação de dependência espacial utilizam apenas os parâmetros de efeito pepita e contribuição. Por outro lado, uma nova metodologia denominada de Índice de Dependência Espacial (IDE) foi proposta por Seidel e Oliveira (2014a) em que esta inclui como parâmetro o alcance e um fator de correção para o modelo. Segundo esses autores o IDE é uma medida mais robusta, em que considera a correlação espacial e todos os parâmetros do semivariograma. O IDE tem como objetivo considerar os aspectos geométricos do semivariograma e - correlograma, abrangendo todas as características da dependência espacial. Destaca-se que o intervalo do IDE varia entre 0 a $100 \%$ (fator de modelo), sendo de 0 a $37,5 \%$ para o modelo esférico, 0 a $31,7 \%$ para o modelo exponencial e de 0 a $50,4 \%$ para o modelo gaussiano.

Salienta-se que a escolha do modelo é uma etapa de suma importância, pois este pode afetar o uso de interpoladores espaciais como a krigagem, interpondo assim na confiabilidade das estimativas, característica essencial para a construção dos mapas temáticos (SCOLFORO et al., 2015). Cabe destacar que, a krigagem propicia a estimativa da variável em estudo para locais que não foram amostrados, em que leva-se em consideração a correlação entre os locais amostrados e a distância entre eles, com variância mínima e sem viés (OLIVEIRA et al., 2006; GUEDES et al., 2012; SEIDEL; OLIVEIRA, 2015).

Assim, o objetivo do presente trabalho foi avaliar o efeito da escolha na seleção de modelos teóricos de semivariograma nas predições volumétricas e estratificação de povoamentos de Eucalyptus sp., comparando diferentes metodologias de mensuração do grau de continuidade espacial.

\section{- Base de dados}

\section{MATERIAL E MÉTODOS}

Os dados deste estudo são provenientes de povoamentos clonais de Eucalyptus sp., com idade variando de 24 a 60 meses e divididos em 50 projetos, situados em três regiões do estado de Minas Gerais, Centro (Curvelo), Noroeste (João Pinheiro) e Norte (Montes Claros). As áreas totais dos povoamentos correspondem à 9.796,6 hectares, sendo $3.197,8$ ha no município de Curvelo, 
3.842,9 ha no município de João Pinheiro e 2.755,9 ha no município de Montes Claros.

O levantamento dos dados se deu a partir de inventários florestais realizados por meio da amostragem sistemática, em que o processamento foi realizado a partir dos estimadores da amostragem casual simples. Para tanto, foram alocadas parcelas circulares com área de $400 \mathrm{~m}^{2}$ considerando intensidade amostral de uma parcela a cada 10 ha.

Nas parcelas amostradas, foram mensurados os diâmetros a 1,30 m de altura do solo (DAP) de todos os indivíduos, a altura total dos 10 primeiros indivíduos, bem como a altura das árvores dominantes de acordo com o conceito de Assman (1970). As coordenadas centrais de todas as parcelas para realização do estudo variográfico também foi anotado para cada uma das parcelas.

Posteriormente as mensurações, procedeu-se com a estimativa da altura para as demais árvores da parcela mediante a aplicação da relação hipsométrica. Por fim, realizou-se a predição de volume para todas as parcelas por meio de uma equação do povoamento.

\section{- Análises Geoestatística}

Visando avaliar o comportamento dos atributos de interesse, realizou-se a análise exploratória dos dados (AED) que possibilita a verificação quanto a presença de tendência e presença de dados discrepantes. Em seguida, efetuou-se o estudo variográfico a partir do ajuste dos semivariogramas experimentais (Equação 1), analisando a estrutura de dependência espacial para cada um dos três projetos avaliados.

$$
\gamma(h)=\frac{1}{2 N(h)} \sum_{i=1}^{N i h}\left[z\left(x_{i}\right)-z\left(x_{i}+h\right)\right]^{2}
$$

Em que $y(h)$ é a semivariância estimada entre pares de pontos; $N(h)$ é o número de pares de valores medidos em $z\left(x_{i}\right), z\left(x_{i}+h\right)$ separados pela distância $h$.

Com o intuito de modelar a semivariância em função da distância, foram ajustados dois modelos teóricos, modelo Esférico (Equação 2) e modelo Exponencial (Equação 3), descritos por Journel e Huijbregts (1978), possibilitando assim a obtenção dos parâmetros dos modelos. Os ajustes foram realizados pelo método da Máxima Verossimilhança $(\mathrm{ML})$ e pelo método dos Quadrados Mínimos Ordinários (OLS), sendo estes implementados por intermédio do pacote geoR (RIBEIRO JÚNIOR ; DIGGLE, 2001) no software R (R CORE TEAM, 2018). A partir dos parâmetros ajustados dos modelos espaciais, determinou-se o grau de Dependência Espacial (DE) e o Índice de Dependência Espacial (IDE).

$$
\gamma(h)=\left\{\begin{array}{l}
0,|h|=0 \\
\tau^{2}+\sigma^{2}\left[1,5 \frac{|h|}{\phi}-0,5 \frac{|h|}{\phi}\right], 0 \leq|h| \leq \phi \\
\tau^{2}+\sigma^{2},|h|>\phi
\end{array}\right.
$$




$$
\gamma(h)=\left\{\begin{array}{l}
0,|h|=0 \\
\tau^{2}+\sigma^{2}\left[1-\exp \left(-\frac{|h|}{\phi}\right)\right],|h| \neq 0
\end{array}\right.
$$

Em que: $\gamma(h)$ é a semivariância estimada entre pares de pontos; $h$ é a distância entre os pares de pontos; $\tau^{2}$ é o efeito pepita; $\sigma^{2}$ é a contribuição; $\emptyset$ é o alcance.

Ainda com a finalidade de auxiliar na escolha do melhor modelo, efetuou-se a análise do grau de dependência espacial em relação a característica volume. Ressalta-se que o grau de dependência espacial (GDE) relaciona o porcentual de variação estruturada em relação ao patamar e foi apresentado por Biondi et al. (1994). Assim, o grau de dependência espacial acima de $75 \%$ é tido como forte, entre 35 e $75 \%$ é considerado médio e abaixo de $35 \%$ como fraco.

\section{- Grau de Dependência Espacial (GDE)}

As metodologias propostas por Biondi et al. (1994) e Cambardella et al. (1994) expressam essencialmente as mesmas informações. Destaca-se que a medida de dependência espacial encontrada no trabalho de Cambardella et al. (1994) é representada pela Equação 4.

$$
G D E_{(\%)}=\left(\frac{\tau^{2}}{\tau^{2}+\sigma^{2}}\right)
$$

Em que $\tau^{2}$ é o efeito pepita; $\sigma^{2}$ é a contribuição.

\section{- Índice de Dependência Espacial (IDE)}

O Índice proposto por Seidel e Oliveira (2014a), que é representado de forma generalizada pela Equação 5 , pode ser utilizado na avaliação de dependência espacial para os modelos de semivariograma exponencial, gaussiano e esférico.

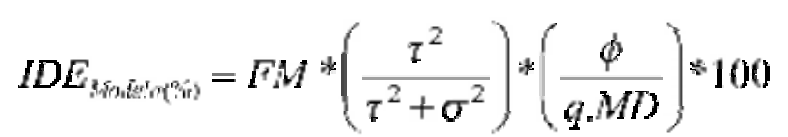

Em que $F M$ é o fator de correção do modelo $(F M=0,375$ para modelo esférico e $F M=0,371$ para modelo exponencial); $\tau^{2}$ é o efeito pepita; $\sigma^{2}$ é a contribuição; $\emptyset$ é o alcance prático; $q \cdot M D$ é $O$ valor correspondente à fração $(q)$ atingida a máxima distância $(M D)$ entre os pontos amostrados.

Quando a relação $\emptyset /(q . M D)$ for maior que 1, essa razão é fixada em 1, assumindo somente valores entre zero e 1. Além disso, quanto maior o valor de $F M$, maior será a força da dependência espacial do modelo. Quando o modelo apresentar o menor valor de fator de modelo é o de efeito pepita puro, em que possui $F M$ zero.

\section{- Classificação da Dependência Espacial}

O grau de dependência espacial para os dois métodos é classificado em forte, moderado e fraca dependência espacial. Quando analisado o IDE, os intervalos de valores do índice variam de acordo com o modelo, sendo entre zero e 37,5\% para o modelo esférico e entre zero e $31,7 \%$ para o modelo exponencial. Os intervalos de classificação para os dois índices são apresentados na Tabela 1. 
TABELA 1 - Classificação da dependência espacial para os dois métodos empregados

\begin{tabular}{cccc}
\hline \multirow{2}{*}{ Método } & \multirow{2}{*}{ Classificação } & \multicolumn{2}{c}{ Modelo } \\
\cline { 3 - 4 } & & Esférico & Exponencial \\
\hline \multirow{2}{*}{ DE $(\%)$} & Moderada & \multicolumn{2}{c}{$>75$} \\
& Entre 35 e 75 \\
& Fraca & \multicolumn{2}{c}{$<35$} \\
\hline \multirow{2}{*}{ IDE $(\%)$} & Forte & $>15$ & $>13$ \\
& Moderada & Entre 7 e 15 & Entre 6 e 13 \\
& Fraca & $>7$ & $>6$ \\
\cline { 2 - 4 }
\end{tabular}

\section{- Comparação dos Índices}

Com o intuito de comparar os índices, foram selecionados três ajustes que apresentaram diferença da classificação dos métodos de estimativa de dependência espacial, sendo uma para cada região avaliada: Curvelo, João Pinheiro e Montes Claros. Baseado nos modelos selecionados procedeu-se a construção de dois mapas de krigagem para cada região, sendo cada um deste gerado com base nos parâmetros do modelo selecionado por cada método.

Os mapas de krigagem foram classificados em cinco classes volumétricas e determinado suas respectivas áreas, gerando o volume por classe volumétrica e total. Por fim, realizou-se análise de correlação dos mapas gerados a partir da seleção de cada modelo no respectivo método de avaliação de dependência espacial.

\section{RESULTADOS E DISCUSSÃO}

De acordo com a classificação pelo índice proposto por Biondi et al. (1994) aproximadamente $60 \%$ dos ajustes foram classificados como forte dependência espacial. Pela classificação do índice de Seidel e Oliveira (2014a) aproximadamente $50 \%$ dos ajustes apresentaram forte dependência espacial, conforme observado na Figura 1.

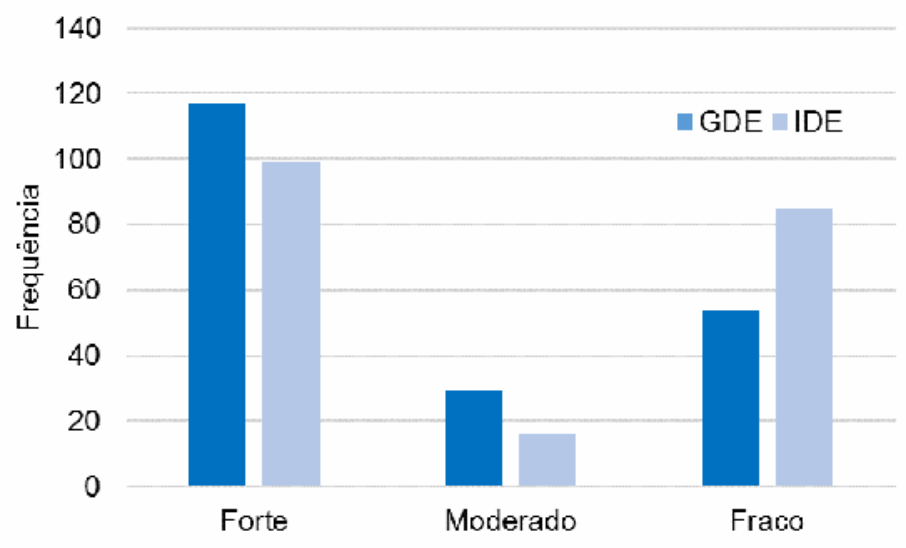


FIGURA 1 - Classificação da dependência espacial nos dois métodos de ajuste e para os dois modelos avaliados.

O índice GDE classificou 117 ajustes como forte dependência espacial, sendo que pelo novo índice (IDE) $40 \%$ dos ajustes mudaria a classificação de forte para fraca dependência espacial, o que afeta diretamente na seleção do ajuste.

De modo geral, 39,5\% das análises geoestatísticas apresentaram alteração da classificação da dependência espacial, conforme observado na Figura 2. Quando comparado os métodos de ajustes, o método dos Mínimos Quadrados Ordinários obteve o maior número de alteração da classificação da dependência espacial em relação ao Método da Máxima Verossimilhança, alterando em aproximadamente $28 \%$ das análises. Por outro lado, os diferentes modelos apresentaram número de alterações próximas.
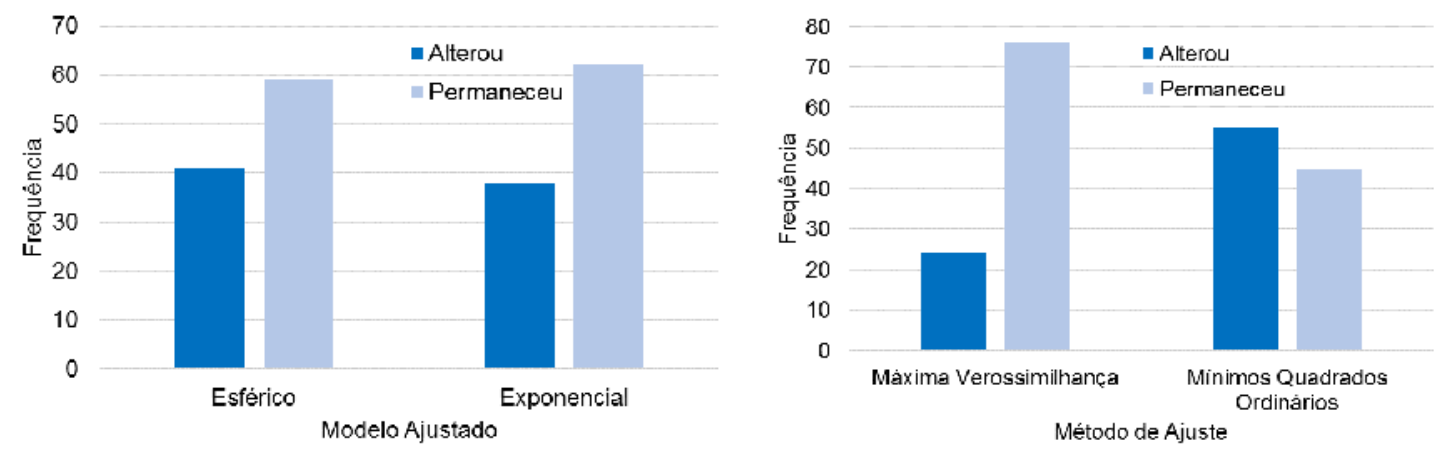

FIGURA 2 - Classificação da dependência espacial nos dois métodos de ajuste e para os dois modelos avaliados.

Ao comparar os modelos em cada um dos métodos de ajuste (Figura 3), observa-se que o ajuste pela ML um número de alterações próximo, enquanto pelo ajuste de OLS, o modelo Esférico apresentou maior variação da classificação.
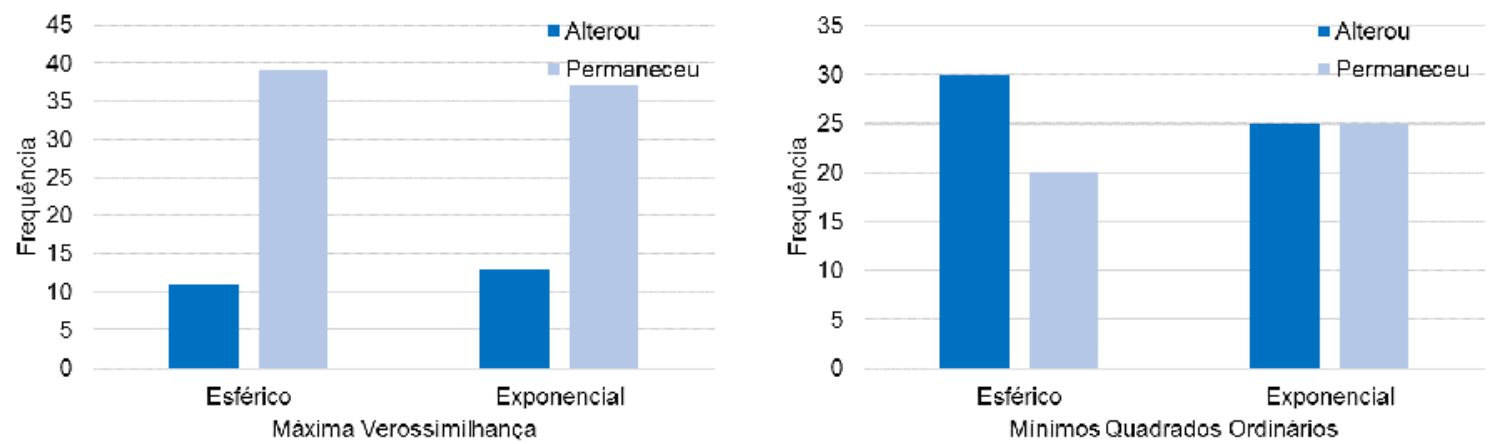

FIGURA 3 - Classificação da dependência espacial para os modelos avaliados e diferentes métodos de ajuste.

Informações no que diz respeito a seleção dos três projetos selecionados, assim como esclarecimentos quanto ao método de ajuste, modelo selecionado e classificação do grau de dependência espacial, estão propostas no Quadro 1. 
QUADRO 1 - Projetos selecionados por região e suas respectivas informações quanto a dependência espacial.

\begin{tabular}{ccccc}
\hline \multirow{2}{*}{ Região } & Método de ajuste & Modelo & GDE & IDE \\
\hline Curvelo & OLS & Exponencial & Forte & Fraca \\
\hdashline & ML & Esférico & Moderada & Forte \\
\hdashline João Pinheiro & OLS & Exponencial & Forte & Fraca \\
\hdashline & ML & Esférico & Moderada & Forte \\
\hdashline Montes Claros & OLS & Exponencial & Forte & Fraca \\
\hline
\end{tabular}

Em que OLS é mínimos quadrados ordinários e ML é a máxima verossimilhança.

De acordo com a Tabela 3, que vem retratando a correlação dos mapas gerados por região e considerando os métodos e modelos selecionados, observa-se que todas as áreas apresentaram valores altos de correlação que vão de 0,98169 (Montes Claros) a 0,99044 (João Pinheiro).

Tabela 2 - Correlação entre os mapas temáticos gerados de acordo as informações do GDE e IDE para cada região de estudo.

\begin{tabular}{cc}
\hline Região & Correlação \\
\hline Curvelo & 0,98885 \\
João Pinheiro & 0,99044 \\
Montes Claros & 0,98169 \\
\hline
\end{tabular}

Atentando-se para os mapas temáticos e considerando que os mesmos obedeceram a um mesmo critério para a elaboração e construção quanto ao número de classes, pode-se inferir que visualmente estes vêm confirmando os valores encontrados pela correlação. A região de Curvelo, que apresentou correlação igual a 0,98885 , apresenta uma pequena diferenciação na área de abrangência no que se refere a quarta e quinta classe (FIGURA 4). Em relação a região de João Pinheiro, pode-se inferir que a transição de uma classe para outra foi praticamente insignificante, apresentando assim uma maior semelhança entre os mapas e justificando a maior correlação obtida. Por fim, os mapas temáticos que caracterizam a região de Montes Claros apresentam um comportamento semelhante ao encontrado para a região de Curvelo, em que o deslocamento da característica de interesse entre as classes está mais acentuado nas últimas classes. 


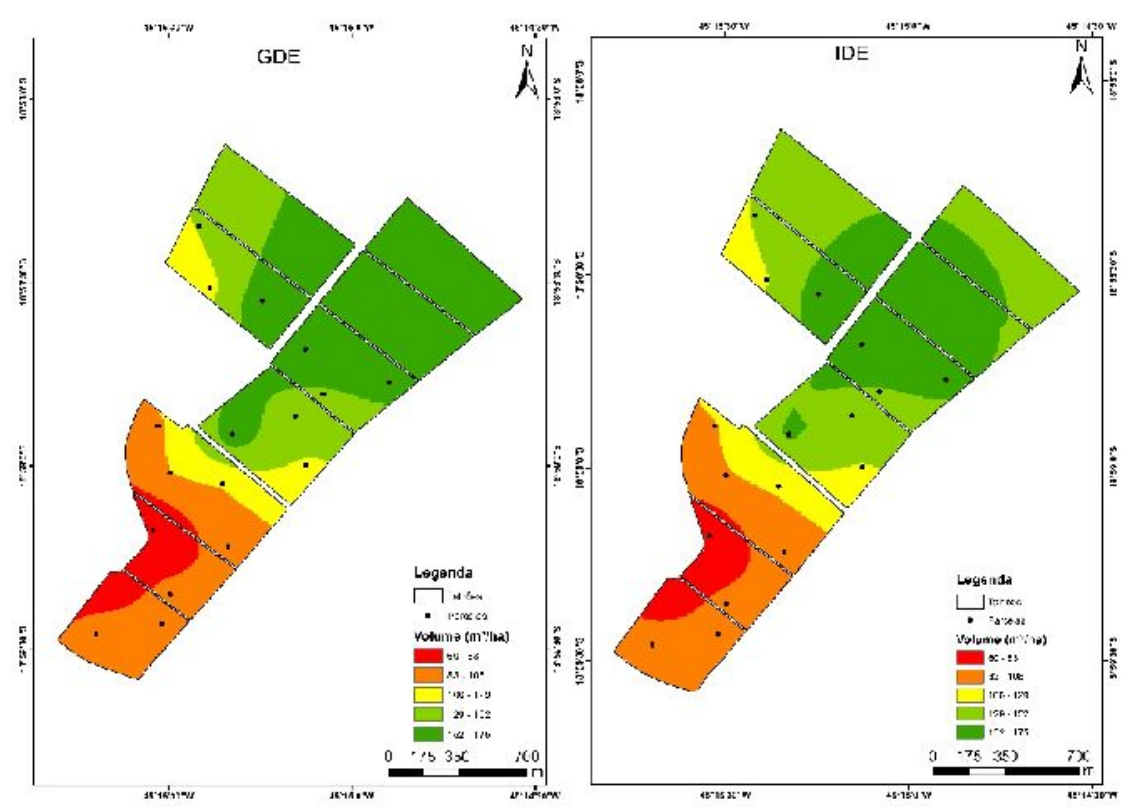

FIGURA 4 - Mapa de krigagem para região de Curvelo de acordo com a seleção dos métodos avaliados.

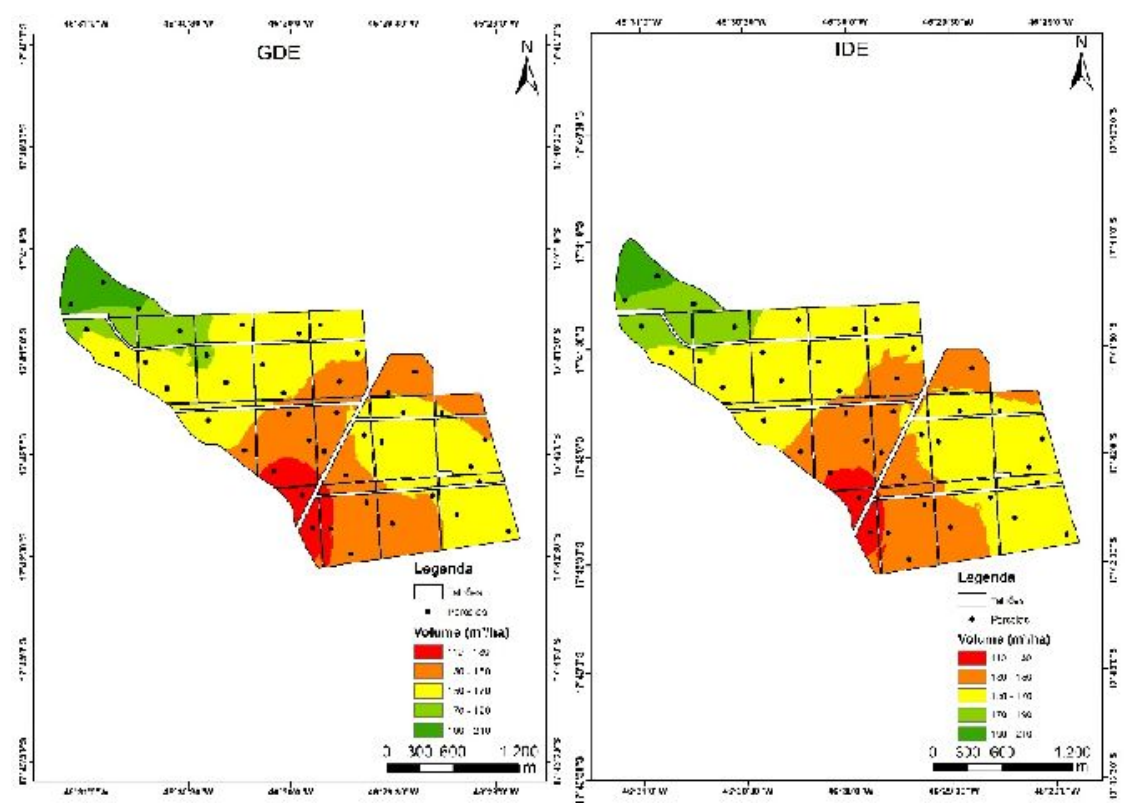

FIGURA 5 - Mapa de krigagem para região de João Pinheiro de acordo com a seleção dos métodos avaliados. 


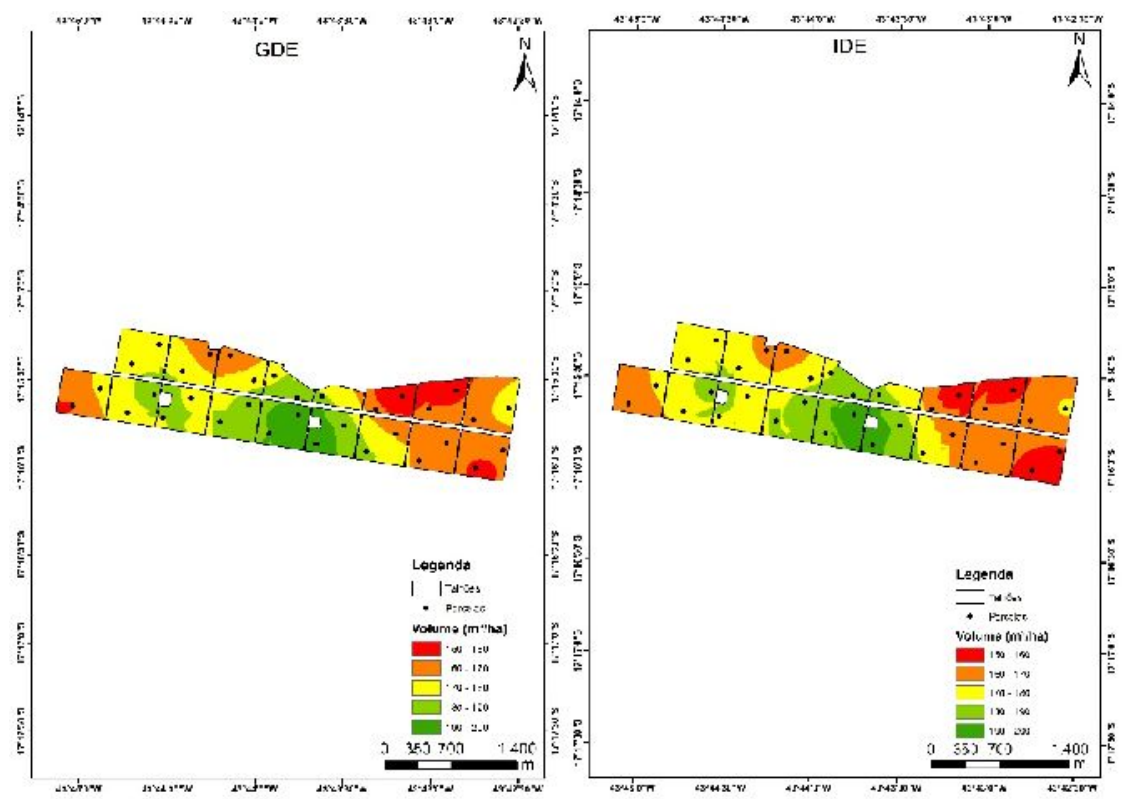

FIGURA 6 - Mapa de krigagem para região de Montes Claros de acordo com a seleção dos métodos avaliados.

A fim de comprovar os resultados encontrados, a Tabela 4 apresenta informações descritivas quanto ao volume médio de cada classe e respectivas áreas e volume de cada metodologia empreendida.

Tabela 3 - Informações quantitativas da área e volume por classe para cada região.

\begin{tabular}{|c|c|c|c|c|c|}
\hline \multirow{2}{*}{ Região } & \multirow{2}{*}{$\begin{array}{c}\text { Volume Médio } \\
\text { da Classe }\end{array}$} & \multicolumn{2}{|c|}{ Área (ha) } & \multicolumn{2}{|c|}{ Volume $\left(\mathrm{m}^{3}\right)$} \\
\hline & & GDE & IDE & GDE & IDE \\
\hline \multirow{5}{*}{ Curvelo } & 71,5 & 14,2 & 12,6 & $1.012,69$ & 903,73 \\
\hline & 94,5 & 41,3 & 42,0 & $3.906,03$ & $3.972,97$ \\
\hline & 117,5 & 19,1 & 19,3 & $2.245,22$ & $2.264,33$ \\
\hline & 140,5 & 41,2 & 68,5 & $5.792,02$ & $9.625,96$ \\
\hline & 163,5 & 90,1 & 63,4 & $14.724,40$ & $10.365,00$ \\
\hline \multirow{5}{*}{$\begin{array}{c}\text { João } \\
\text { Pinheiro }\end{array}$} & 120 & 28,7 & 20,0 & $3.439,13$ & $2.398,80$ \\
\hline & 140 & 155,6 & 151,9 & $21.783,10$ & $21.262,21$ \\
\hline & 160 & 253,1 & 266,2 & $40.493,65$ & $42.594,82$ \\
\hline & 180 & 37,5 & 46,4 & $6.745,06$ & $8.350,02$ \\
\hline & 200 & 24,8 & 15,2 & $4.952,14$ & $3.036,61$ \\
\hline \multirow{5}{*}{$\begin{array}{l}\text { Montes } \\
\text { Claros }\end{array}$} & 155 & 30,3 & 34,8 & $4.699,50$ & $5.388,21$ \\
\hline & 165 & 112.0 & 114.5 & 18.484 .81 & 18.896 .25 \\
\hline & 175 & 119,4 & 119,4 & $20.891,71$ & $20.900,05$ \\
\hline & 185 & 67,5 & 66,9 & $12.486,00$ & $12.381,25$ \\
\hline & 195 & 28,6 & 22,2 & $5.582,59$ & $4.326,33$ \\
\hline
\end{tabular}

Observou-se que para os três projetos avaliados, em três diferentes regiões, houve grandes diferenças na estimativa de volume. Na região de Curvelo o mapa gerado baseado na escolha feita pelo IDE estimou aproximadamente $550 \mathrm{~m}^{3}$ a mais do que a estimativa realizada baseada pelo GDE. Tal comportamento é semelhante ao encontrado na região de Montes Claros, onde o IDE estimou aproximadamente $250 \mathrm{~m}^{3}$ do que o GDE. Já para a região de João Pinheiro, o GDE estimou um volume de aproximadamente $230 \mathrm{~m}^{3}$ a mais que o IDE. 
Seidel e Oliveira (2014b) descrevem o IDE como um índice mais completo quando comparado aos índices de Biondi et al. (1994) e de Cambardella et al., (1994). Tal consideração está atrelada ao fato que o IDE utiliza, além do efeito pepita e contribuição, todos os parâmetros dos modelos da dependência espacial e um fator de modelo. Ademais, os autores relatam que esse fator de modelo deve ser ponderado, uma vez que por se tratar de variáveis observadas na natureza, elas não conseguem atingir $100 \%$ de dependência espacial.

O direcionamento quanto a tomada de decisão acerca da viabilidade de empreendimentos florestais é realizada a partir dos resultados dos inventários, sendo estes instrumentos básicos utilizados para avaliar estatisticamente as reais potencialidades e capacidades produtivas dos recursos florestais de uma área (BARROS et al., 2015).

Assim, a utilização de uma base de dados robusta, com 50 projetos florestais, juntamente com 0 uso de mais informações do semivariograma experimental, corroboram para o uso do IDE para descrever o grau de dependência espacial comparado aos índices tradicionalmente utilizados. Portanto, com a utilização dos outros índices pode-se cometer equívocos na classificação, ocasionando seleções errôneas dos modelos, inferindo na qualidade das predições através de interpolador espacial (krigagem) e na inferência da variabilidade da variável regionalizada em estudo. Diante disso, fica evidenciada a importância da adoção do novo índice (IDE) para caracterizar a estrutura de continuidade espacial, mostrando-se um bom índice de classificação.

\section{CONCLUSÃO}

Aproximadamente $40 \%$ dos ajustes classificados como forte dependência espacial pelo GDE mudou a classificação para fraca dependência espacial pelo IDE, o que acarretaria diferenças na interpolação espacial.

O método IDE foi o mais recomendado para descrever a dependência espacial, devido contemplar todos os parâmetros do semivariograma, bem como um fator de correção para cada modelo, apresentando como um índice mais robusto. Uma vez que o GDE apresenta fragilidade por não levar em consideração o parâmetro alcance e o modelo ajustado.

\section{AGRADECIMENTOS}

Os autores agradecem à Universidade Federal de Lavras (UFLA) pela estrutura e disponibilidade dos recursos necessários para a pesquisa.

\section{REFERÊNCIAS}

ACERBI JÚNIOR, F.W.; SILVEIRA, E.M.O.; MELLO, J.M.; MELLO, C.R.; SCOLFORO, J.R.S. Change detection in Brazilian Savannas using semivariograms derived from NDVI images. Revista Ciência e Agrotecnologia, v. 39, n.2, p. 103109, 2015. Disponível em: < http://www.scielo.br/pdf/cagro/v39n2/1981-1829-cagro39-02-00103.pdf>. doi: 10.1590/S1413-70542015000200001

ASSMANN, E. The principles of forest yield study. New York: Pergamon Press, 506p, 1970.

BARROS, B.S.X.; GUERRA, S.P.S.; BARROS, Z.X.; CATITA, C.M.S.; FERNANDES, J.C.C. Uso de imagens de satélite para cálculo de volume em floresta de eucalipto 
no município de Botucatu/SP. Revista Energia na Agricultura, v. 30, n. 1, p. 60-67, 2015. Disponível em: < https://doi.org/10.17224/EnergAgric.2015v30n1p60-67 >. doi: 10.17224/EnergAgric.2015v30n1p60-67

BINOTI, M.L.M.; BINOTI, D.H.B.; LEITE, H.G.; GARCIA, S.L.R.; FERREIRA, M.Z., et al. Redes neurais artificiais para estimação do volume de árvores. Revista Árvore, v. 38, n. 2, p. 283-288, 2014. Disponível em: < http://dx.doi.org/10.1590/S010067622014000200008>. doi: 10.1590/S0100-67622014000200008

BIONDI, F.; MYERS, D.E.; AVERY, C.C. Geostatistically modeling stem size and increment in an old-growth forest. Canadian Journal of Forest Research, v. 24, p. 1354-1368, 1994. Disponível em: < https://doi.org/10.1139/x94-176>. doi: $10.1139 / \times 94-176$

CAMBARDELLA, C.A.; MOORMAN, T.B.; NOVAK, J.M.; PARKIN, T.B.; KARLEN, D.L., et al. Field-Scale Variability of Soil Properties in Central lowa Soils. Soil Science Society of America Journal, v. 58, n. 5, p. 1501-1511, 1994. Disponível em: < $\quad$ https://www.researchgate.net/publication/43269985_FieldScale_Variability_of_Soil_Properties_in_Central_lowa_Soils $>$. doi: 10.2136/sssaj1994.03615995005800050033x

GORGENS, E.B.; LEITE, H.G.; GLERIANI, J.M.; SOARES, C.P.B.; CEOLIN,A. Influência da arquitetura na estimative de volume de árvores individuais por meio de redes neurais artificiais. Revista Árvore, v.38, n.2, p.289-295, 2014. Disponível em: < https://www.redalyc.org/pdf/488/48831289009.pdf>. doi: 10.1590/S010067622014000200009

GUEDES, I.C. de L.; MELLO, J.M. de; MELLO, C.R. de; OLIVEIRA, A.D. de; SILVA, S.T. da, et al. Técnicas Geoestatísticas e Interpoladores Espaciais na Estratificação de Povoamentos de Eucalyptus sp. Ciência Florestal, Santa Maria, v. 22, n. 3, p. 541-550, 2012. Disponível em: <http://dx.doi.org/10.5902/198050986621>. doi: $10.5902 / 198050986621$

JOURNEL, A.G.; HUIJBREGTS, J.C.H. Mining geostatistics. Virginia: Universidade da Virginia, 1978.

LEAL, F.A.; MIGUEL, E.P.; MATRICARDI, E.A.T.; PEREIRA, R.S. Redes neurais artificiais na estimativa de volume em um plantio de eucalipto em função de fotografias hemisféricas e número de árvores. Revista Brasileira de Biometria, v. 33, n. 2, p. 233-249, 2015. Disponível em: < http://jaguar.fcav.unesp.br/RME/fasciculos/v33/v33_n2/A8_Fabricio_Eder_Eraldo.pdf $>$.

LUNDGREN, W.J.C.; SILVA, A.A. da J.; FERREIRA, L.C.R. Influência do Tipo de Amostragem na Estimativa de Volume de Madeira de Eucalipto por Krigagem. Revista Floresta e Ambiente, Seropédica-RJ, v. 23, n. 4, p. 511-523, 2016. Disponível em: < http://dx.doi.org/10.1590/2179-8087.136315>. doi: 10.1590/21798087.136315 
LUNDGREN, W.J.C.; SILVA, J.A.S.; FERREIRA, R.L.C. Estimação de volume de madeira de eucalipto por cokrigagem, krigagem e regressão. Cerne, v. 21, n. 2, p. 243-250, 2015. Disponível em: < http://www.scielo.br/pdf/cerne/v21n2/2317-6342cerne-21-02-00243.pdf>. doi: 10.1590/01047760201521021532

MELLO, C.R. de; VIOLA, M.R.; MELLO, J.M. de. Continuidade espacial de chuvas intensas no Estado de Minas Gerais. Ciência e Agrotecnologia, Lavras, v. 32, n. 2, p. 532-539, 2008. Disponível em: < http://dx.doi.org/10.1590/S141370542008000200029 >. doi: 10.1590/S1413-70542008000200029

ODA-SOUZA, M.; BARBIN, D.; RIBEIRO JÚNIOR, P.J.; STAPE, J.L. Aplicação de métodos geoestatísticos para identificação de dependência espacial na análise de dados em um ensaio de espaçamento florestal em delineamento sistemático tipo leque. Revista Árvore, Viçosa, v. 32, n. 3, p. 499-509, 2008. Disponível em: <http://dx.doi.org/10.1590/S0100-67622008000300011>. doi: 10.1590/S010067622008000300011

OLIVEIRA, L.T.; FERREIRA, M.Z.; CARVALHO, L.M.T.; FERRAZ FILHO, A.C.; OLIVEIRA, T.C.A., et al. Determinação do volume de madeira em povoamento de eucalipto por escâner a laser aerotransportado. Pesquisa agropecuária brasileira. v. 49, n.9, p. 692-700, 2014. Disponível em: <http://dx.doi.org/10.1590/S0100204X2014000900005>. doi: 10.1590/S0100-204X2014000900005

OLIVEIRA, M.C.N.; BARBIN, D.; SILVA, E.F.; MACEDO, J.R.; CAPECHE, C.L., et al.; Estimação dos parâmetros de uma função de covariância no estudo da variabilidade espacial do teor de cálcio no solo. Revista de Matemática e Estatística, Jaboticabal, v. 24, n. 4, p. 117-132, 2006. Disponível em: < http://jaguar.fcav.unesp.br/RME/fasciculos/v24/v24_n4/A8_Cristina.pdf >.

R CORE TEAM. R: a language and environment for statistical computing. $\mathbf{R}$ Foundation for Statistical Computing, Vienna, Austria. 2018. Disponível em: $<U R L$ https://www.Rproject.org/>.

RIBEIRO JÚNIOR, P.J; DIGGLE, P.J. GeoR: a package for geostatistical analysis. RNEWS, v.1, n.2, p.15-18, 2001. Disponível em: < http://citeseerx.ist.psu.edu/viewdoc/download?doi=10.1.1.464.3971\&rep=rep1\&type= pdf $>$.

SANTOS, M.C. dos; ROVEDA, M.; ZANON, M.L.B.; FIGUEIREDO FILHO, A.; ROIK, M., et al. Inventário florestal utilizando técnicas de silvicultura de precisão em povoamentos de Eucalyptus grandis hill ex maiden. Revista Floresta e Ambiente, Seropédica-RJ, v. 24, 2017. Disponível em: < http://dx.doi.org/10.1590/21798087.082714>. doi: 10.1590/2179-8087.082714

SCOLFORO, H.F.; SCOLFORO, J.R.S.; MELLO, C.R.; MELLO, J.M.; FERRAZ FILHO, A.C. Spatial distribution of aboveground carbon stock of the arboreal vegetation in Brazilian biomes of Savanna, Atlantic Forest and Semi-Arid Woodland. Plos One, v. 10, n. 6, p. 1-20, 2015. Disponível em:

<https://doi.org/10.1371/journal.pone.0128781>. doi:10.1371/journal.pone.0128781 
SEIDEL, E.J.; OLIVEIRA, M.S. De. Definição de áreas de dependência espacial em semivariogramas. Revista da Estatística, v. 3, n. 3, p. 348-352, 2014b. Disponível em: < http://www.cead.ufop.br/jornal/index.php/rest/article/viewFile/595/499 >.

SEIDEL, E.J.; OLIVEIRA, M.S. de. Medidas de dependência espacial baseadas em duas perspectivas do semivariograma paramétrico. Ciência e Natura, Santa Maria, v. 37, n. 4, p. 20-27, 2015. Disponível em: < http://dx.doi.org/10.5902/2179460X18049>. 10.5902/2179-460X18049

SEIDEL, E.J.; OLIVEIRA, M.S. de. Novo Índice Geoestatístico para a Mensuração da Dependência Espacial. Revista Brasileira de Ciência do Solo, Viçosa, v. 38, n. 3, p. 699-705, 2014a. Disponível em: < http://dx.doi.org/10.1590/S010006832014000300002>. doi: 10.1590/S0100-06832014000300002 